\title{
Facile synthesis of various 2-substituted-4-(2-pyridyl) benzopyran analogues as target potassium channel opener
}

\author{
A. K. Tripathi,* Debaraj Mukherjee, S. Koul, and Subhash C. Taneja \\ Indian institute of integrative medicines (CSIR), Canal Road, Jammu-180001, India \\ E-mail: tripathitripathi@rediffmail.com
}

\begin{abstract}
A series of 2-substituted-4-(2-pyridyl)benzopyran analogues 6a-e have been prepared in 20-34\% overall yields by the reductive condensation of 2-substituted-3,4-dihydro-2H-1-benzopyran derivatives 5a-e with pyridine, as a key step.
\end{abstract}

Keywords: Benzopyran, acetophenone, pyridine, $\mathrm{Al} / \mathrm{HgCl}_{2}$, potassium channel openers

\section{Introduction}

4-substituted benzopyran derivatives constitute a class of compounds with application as potassium channel openers, which regulate changes in the intracellular level of adenosine triphosphate (ATP). These channels are closed when intracellular ATP levels are elevated and opened when the level declines, thus linking membrane potential to the metabolic state of the cell. ${ }^{1}$ Opening them allows the passage of potassium ions out of the cell, causing transmembrane hyperpolarization which in turn reduces intracellular calcium concentration by a blocking function of voltage-dependent calcium channels and inhibiting intracellular calcium release, resulting in smooth muscle relaxation and antispasmodic action. ${ }^{2}$ The use of potassium channel openers $^{3-13}$ may therefore, be valuable in treating disorders caused by smooth muscle contraction, such as hypertension, angina pectoris, asthma, ${ }^{14}$ urinary incontinence, ${ }^{15}$ and baldness. ${ }^{16}$ Additionally, these agents may provide the cells a measure of protection against ischemia, independent of their vasodilating actions, ${ }^{17}$ and have antilipidemic effects, lowering low density lipoprotein (LDL) cholesterol and triglycerides while increasing high density lipoprotein (HDL) cholesterol. $^{18}$ 
<smiles>CC1(C)Oc2ccc(C#N)cc2[C@@H](N2CCCC2=O)[C@@H]1O</smiles>
lemakalim[(-)-(3S,4R)-cromakalim $]^{19}$<smiles>CC1(C)Oc2ccc(OC(F)(F)F)cc2[C@H](N2Cc3ccccc3C2=O)[C@@H]1O</smiles>
(-)-celikalim ${ }^{22}$

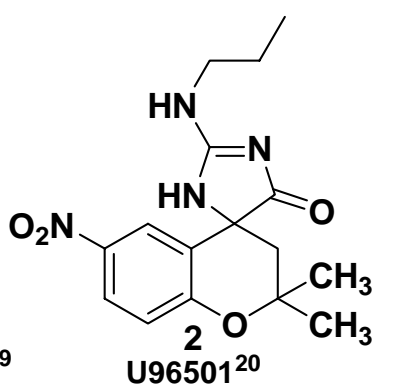<smiles>CC1(C)Oc2ccc(C#N)cc2[C@@H](Nc2ccccc2)[C@@H]1O</smiles>

SR46142A ${ }^{23}$<smiles>CC(C)(C)O[R6](=O)(O)C1(C)C=C(n2ccccc2=O)c2cc(C#N)ccc2O1</smiles><smiles>[R6][R16]#[R16]</smiles>

Figure 1. 4-Substituted benzopyran derivatives ${ }^{19-24}$ as potassium channel openers.

There are several prototypes of this class of compound (Figure 1) all having a common structural moiety i.e, 4-substituted benzopyran 1-6. ${ }^{19-24}$ As part of a program to develop new compounds we decided to prepare 4-(2-pyridyl)-4-hydroxybenzopyran and substituted derivatives as target potassium channel openers.

There are two broad approaches for the synthesis of 4-substituted benzopyrans. The first approach $^{18,25}$ deals with the opening of an epoxide at the 3,4-double bond of the benzopyran nucleus (Scheme 1a) by nucleophilic attack of electron rich heterocycles in the presence of base and dehydration to afford 4-substituted chromenes.

(a)<smiles>[R]c1ccc(O)c(C(C)=O)c1</smiles>

(b)<smiles></smiles><smiles>O=[N+]([O-])c1ccc2c(c1)C(c1ccccn1)=CC(C(F)(F)F)(C(F)(F)F)O2</smiles>

Scheme 1. Literature method for synthesis of 4-hetero-substituted chromenes. 
Another strategy ${ }^{26}$ involves reaction of benzopyran-4-one with $\mathrm{Tf}_{2} \mathrm{O}$ followed by concominent attack of organozinc compounds to displace the resulting triflate affording 4pyridyl benzpyran analogues (Scheme 1b).

Both the approaches suffer from poor yields and multiple reaction steps. In particular, the second approach requires the presence of electron withdrawing groups such as trifluoromethyl group at the 2-position of the benzopyran ring. Thus, development of a more flexible and direct approach for the construction of a substituted cromakalim is highly desirable considering the potential medicinal value. In continuation of our recent work ${ }^{27}$ towards the development of novel protocols for the synthesis of substituted benzopyrans for the exploration of potentially biologically active leades, we now report synthesis of various 2-substituted-4-(2-pyridyl) benzopyrans using aluminium amalgam as catalyst in reductive condensation of 2-substituted$2 \mathrm{H}$-1-benzopyran derivatives with pyridine as a key step.

\section{Results and Discussion}

For the synthesis of 2-substituted-7-hydroxy-benzopyran, the Tripathi-Taneja ${ }^{28}$ procedure, a modified general procedure for the preparation of benzopyrans, was chosen (Scheme 2).

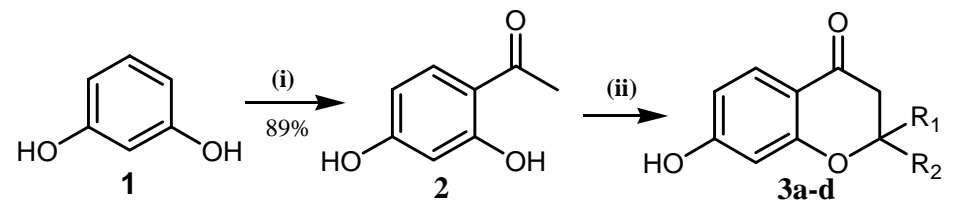

Reagents and conditions: (i) $\mathrm{ZnCl}_{2}, \mathrm{CH}_{3} \mathrm{COOH}$, heat; (ii) pyrrolidine, ketone substrate, benzene, reflux.

Scheme 2. Synthesis of 2-substituted-7-hydroxy-chromanones.

The 2-substituted-7-hydroxy-chromanones were prepared by the base catalysed condensation of substituted acetophenones with carbonyl compounds to give 2,2-disubstituted-7-hydroxybenzopyran-4-ones 3a-d (93-98\%). As anticipated, yields in the condensation reaction decreased with sterically more demanding ketones and the highest yield was observed with acetone as carbonyl partner (Table 1) 
Table 1. Condensation of acetophenones with carbonyl compounds

\begin{tabular}{|c|c|c|c|c|c|}
\hline Entry & Substrate & Product & $\mathrm{R}^{1}+\mathrm{R}^{2}$ & $\mathrm{R}^{3}$ & $\begin{array}{l}\text { Yield } \\
(\%)\end{array}$ \\
\hline 1 & 2 & 3a & & $\mathrm{H}$ & 98 \\
\hline 2 & 2 & $3 b$ & & $\mathrm{H}$ & 92 \\
\hline 3 & 2 & 3c & & $\mathrm{H}$ & 86 \\
\hline 4 & 2 & $3 d$ & & $\mathrm{H}$ & 83 \\
\hline
\end{tabular}

Methylation of the phenolic group afforded 7-methoxy derivatives 4a-d. In order to investigate the effect of substituents in the reductive condensation reaction, $4 \mathbf{d}$ was subjected to nitration with silver nitrate and $\mathrm{Tf}_{2} \mathrm{O}$ in dichloromethane to afford $4 \mathbf{e}$ in $79 \%$ yield. To introduce a pyridyl group at $\mathrm{C}$-4, we resorted to the heterogeneous reductive condensation method reported by Emmert and Asendorf, ${ }^{29}$ with some modification. Thus the reaction was carried out by condensation of benzopyran with pyridine in the presence of aluminium amalgam $\left[\mathrm{Al} / \mathrm{HgCl}_{2}\right]$ to afford the desired 4-(2-pyridyl)-substituted benzopyrans 5a-e (Scheme 3).

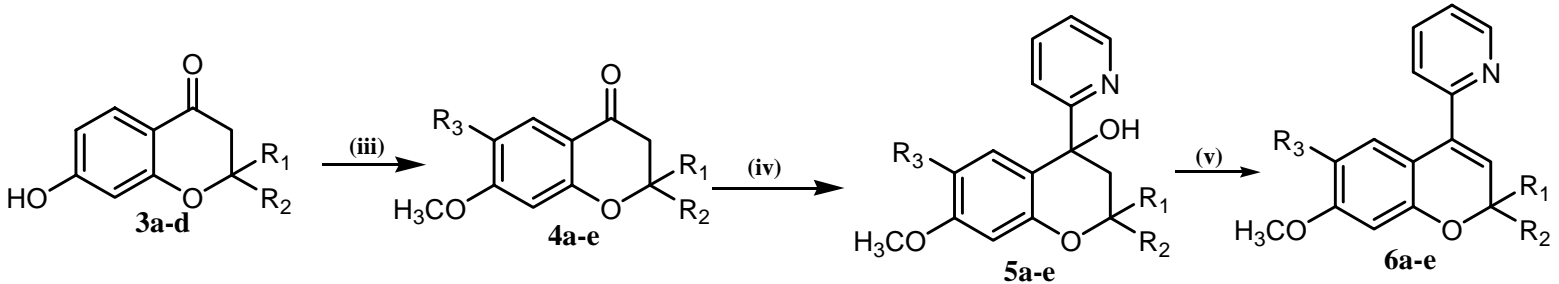

Reagents and conditions: (iii) $\mathrm{CH}_{3} \mathrm{I}$, acetone, $\mathrm{K}_{2} \mathrm{CO}_{3}$, reflux; (iv) i. $\mathrm{AgNO}_{3}$, TFAA, DCM; ii, pyridine, $\mathrm{HgCl}_{2}$-Al, reflux; (vi) $p$-TSA, benzene, reflux.

Scheme 3. Synthesis of 4-(2-pyridyl)-4-hydroxy 3,4-dihydro-benzopyran and 4-(2-pyridyl)-2- $H$ benzopyrans.

The 4-(2-pyridyl) chroman derivatives were identified by NMR spectroscopy, e.g. in the ${ }^{1} \mathrm{H}$ NMR spectrum of compound 5a, besides the signals for pyridine protons which appeared downfield at $\delta 6.99,7.25,7.60$, and 8.53 , those for the methylene proton (H3) appeared as two doublets at $\delta 2.13 \& 2.19$. The other signals appeared at their anticipated positions. 
Table 2. Synthesis of 4-(2-pyridyl) 4-hydroxy-3,4-dihydrobenzopyrans from benzopyran-4-one

\begin{tabular}{|c|c|c|c|c|c|}
\hline Entry & Substrate & Product & $\mathrm{R}^{1}+\mathrm{R}^{2}$ & $\mathrm{R}^{3}$ & Yield (\%) \\
\hline 1. & 3a & $4 a$ & & $\mathrm{H}$ & 99 \\
\hline 2. & $3 b$ & $4 b$ & & $\mathrm{H}$ & 97 \\
\hline 3. & 3c & 4c & & $\mathrm{H}$ & 97 \\
\hline 4. & 3d & $4 d$ & & $\mathrm{H}$ & 97 \\
\hline 5. & $4 a$ & $4 e$ & & $\mathrm{NO}_{2}$ & 79 \\
\hline 6. & $4 a$ & $5 a$ & & $\mathrm{H}$ & 42 \\
\hline 7. & $4 \mathrm{~b}$ & $5 b$ & & $\mathrm{H}$ & 40 \\
\hline 8. & $4 c$ & $5 c$ & & $\mathrm{H}$ & 40 \\
\hline 9. & $4 d$ & $5 d$ & & $\mathrm{H}$ & 39 \\
\hline 10. & $4 e$ & $5 e$ & & $\mathrm{NO}_{2}$ & 33 \\
\hline 11. & $5 a$ & $6 a$ & & $\mathrm{H}$ & 97 \\
\hline 12. & $5 b$ & $6 b$ & & $\mathrm{H}$ & 96 \\
\hline 13. & $5 c$ & 6c & & $\mathrm{H}$ & 94 \\
\hline 18. & $5 d$ & $6 \mathrm{~d}$ & & $\mathrm{H}$ & 93 \\
\hline 14. & $5 e$ & $6 e$ & & $\mathrm{NO}_{2}$ & 89 \\
\hline
\end{tabular}

The structure corroborated by its mass spectrum which showed the $\mathbf{M}^{+}$peak at $m / z 286$ and characteristic fragment peaks at $m / z 268\left(\mathrm{M}^{+}-\mathrm{H}_{2} \mathrm{O}\right)$ and $m / z 189\left(\mathrm{M}^{+}\right.$-pyridine). The structure was further confirmed by its ${ }^{13} \mathrm{C}$-NMR spectrum showing the presence of a quaternary carbon signal at $\delta 75.5$ for C-4. It was observed that electron withdrawing group at C-6 reduced the yield of the coupling reaction to some extent. 
Dehydration of compounds 5a-e with $p$-TSA afforded the desired 4-(2-pyridyl)chromans 6ae in good yields. The formation of dehydration products were confirmed by the disappearance of C-3 methylene resonances at $\delta$ 2.2-2.7 and the appearance of an $\mathrm{H}-3$ olefinic proton signal at $\delta$ 5.80-6.30.

In summary: a series of 2-(7-methoxy-2H-cromen-4-yl)pyridines 6a-e have been prepared by reductive condensation of 2-substituted-3,4-dihydro-2H-1-benzopyrans 5 with pyridine. The structures of all new products have been confirmed by elemental analysis and spectral studies (IR, ${ }^{1} \mathrm{H}$ NMR and Mass Spectrometry). Further biological studies, e.g. cytoxicity, antihypertensive, potassium channel openers, etc of short listed compounds are in progress and will be reported in due course.

\section{Experimental Section}

General. Solvents and other chemicals were of reagent grade and were used without further purification. Laboratory grade solvents were purified and dried by reported methods. All melting points were determined in capillary tubes on a Buchi technical apparatus (BUCHI-510) and are uncorrected. NMR spectra were obtained on Bruker Supercon $200 \mathrm{MHz}$ and $500 \mathrm{MHz}$ instruments and are expressed in $\delta$ values downfield from tetramethylsilane (TMS) as the internal standard. Mass spectra were recorded with a JEOL MS-D 300 mass spectrometer. IR spectra (KBr pellet or neat sample) were recorded on Perkin Elmer-377 and Shimadzu IR-435 spectrophotometers. Column chromatography was performed on silica gel (100-200 mesh) and TLC on silica gel $60 \mathrm{~F}_{254}$ (Merck). For the visualization of spots either UV or iodine vapour or $10 \%$ aqueous sulfuric acid containing $2 \%$ ceric ammonium sulfate or $5 \%$ ethanolic solution of 2,4-dinitrophenylhydrazine was used. Atomic Absorption spectroscopy of all final compounds were performed in Perkin Elmer A Analyst 800 and below detection level (BDL) of $\mathrm{Hg}^{2+}$ ion observed in the range $0.001-0.0006 \%$ in all cases.

\section{Synthesis of 2-substituted-4-hydroxy-3,4-dihydro-4-(2-pyridyl)-2H-1-benzopyran 5a-e} General procedure

To a mixture of $\mathrm{HgCl}_{2}$ (0.037 mole) and freshly prepared aluminium powder heated at $120{ }^{\circ} \mathrm{C}$ for 20 minutes was added a mixture of pyridine $(1.20 \mathrm{ml})$ and $5(0.0080 \mathrm{~mol})$ with vigorous stirring and the contents were refluxed for $1 \mathrm{~h}$. The reaction mixture was cooled and then poured into $6 \mathrm{~N}$ $\mathrm{NaOH}$ solution $(20 \mathrm{ml})$ with vigorous stirring. The aqueous layer was extracted with ethyl acetate. The organic layer was washed with water and finally distilled to give an oily residue. The crude product was chromatographed on silica gel column using petroleum ether:ethyl acetate (80:20) as eluent to give $\mathbf{5}$ as a semisolid. 


\section{Synthesis of 2-substituted-3,4-dihydro-4-(2-pyridyl)-2H-1-benzopyrans 6a-e. General procedure}

To a solution of $5(0.01 \mathrm{~mol})$ in benzene $(50 \mathrm{ml})$ was added $p$-toluenesulfonic acid $(0.2 \mathrm{~g})$. The mixture was heated at reflux temperature for $2 \mathrm{~h}$ and then cooled to room temperature. The contents of the reaction mixture were extracted with ethyl acetate $(3 \times 50 \mathrm{ml})$; the organic layer was washed with brine $(80 \mathrm{ml})$, dried over sodium sulfate, and concentrated on a rotary evaporator under reduced pressure to give a brick red gummy residue, which on chromatography over a silica gel column using petroleum ether-ethyl acetate (80:20) as eluent yielded $\mathbf{6}$ as a gummy mass.

\section{2,2-Dimethyl-4-hydroxy-7-methoxy-3,4-dihydro-4-(2-pyridyl)-2H-1-benzopyran}

5 a. Analysis : found C 70.21, H 6.63, N 4.70\%; $\mathrm{C}_{17} \mathrm{H}_{19} \mathrm{NO}_{3}$ requires C 71.50, H 6.71, N 4.91\%. IR: 3350, 2900, 1620, 1585, 1500, 1440, 1385, 1370, 1340, 1260, 1240, 1200, 1140, 1110, 1080, 1030, 980, 940, 860, 840, 785, 760, $740 \mathrm{~cm}^{-1},{ }^{1} \mathrm{H}$ NMR (200 MHz, $\left.\mathrm{CDCl}_{3}\right): \delta 1.46$ (6H, s, $2 \mathrm{xCH}_{3}$ ), 2.49 (2H, s, $\left.\mathrm{CH}_{2}\right), 3.75$ (3H, s, $\left.\mathrm{OCH}_{3}\right), 6.43(2 \mathrm{H}, \mathrm{m}, 6-\mathrm{H} \& 8-\mathrm{H}), 6.67(1 \mathrm{H}, \mathrm{d}, J=8.5 \mathrm{~Hz}, 5-\mathrm{H}), 6.99$ (1H, dd, $J=2.2 \&$ 8.2Hz, 3H-Py), 7.25 (1H, m, 5H-Py), 7.60 (1H, m, 4H-Py), 8.53 (1H, d, $J=4.4$ $\mathrm{Hz}, 6 \mathrm{H}-\mathrm{Py}) .{ }^{13} \mathrm{C}$ NMR $\left(50 \mathrm{MHz}, \mathrm{CDCl}_{3}\right): \delta 25.7\left(\mathrm{CH}_{3}\right), 25.8\left(\mathrm{CH}_{3}\right), 51.0(\mathrm{C}-3), 55.5\left(\mathrm{OCH}_{3}\right)$, 71.0 (C-2), 75.5 (C-4), 102.0 (C-8), 108.8 (C-6), 119.5 (C-9), 121.6 (C-5), 122.5 (C-5 Py), 130.9 (C-3 Py), 137.5 (C-4 Py), 146.9 (C-2 Py), 155.5 (C-10), 160.8 (C-7), 165.7 (C-6 Py). MS m/z (\%) $285\left(\mathrm{M}^{+}\right)$: 285 (2), 284 (27), 268 (22), 253 (25), 232 (35), 211 (100), 196 (27), 191 (6), 189 (11), 160 (76), 143 (13), 116 (25), 89 (65), 63 (26).

2,2-Dimethyl-7-methoxy-4-(2-pyridyl)-2H-1-benzopyran 6a. Analysis for $\mathrm{C}_{17} \mathrm{H}_{17} \mathrm{NO}_{2}$ (found C 77.81, H 6.39, N 5.08\%, requires C 77.38, H 6.41, N 5.24\%). IR: 3350, 2900, 1610,1600, 1500, 1460, 1439, 1380, 1360, 1290, 1280, 1200, 1140, 1140, 1120, 1040, 985, 895, 840, 800, 780, 740 $\mathrm{cm}^{-1}{ }^{1} \mathrm{H}$ NMR (200 MHz, $\left.\mathrm{CDCl}_{3}\right): \delta 1.49\left(6 \mathrm{H}, \mathrm{s}, 2 \mathrm{xCH}_{3}\right), 3.77\left(3 \mathrm{H}, \mathrm{s},-\mathrm{OCH}_{3}\right), 5.80$ $(1 \mathrm{H}, \mathrm{s}, 3-\mathrm{H}), 6.47(1 \mathrm{H}, \mathrm{d}, J=8.5$ \& 2.Hz, 6-H \& 8-H), 7.14 (1H, dd, $J=2.2 \& 8.2 \mathrm{~Hz}, 3 \mathrm{H}-\mathrm{Py})$, $7.18(1 \mathrm{H}, \mathrm{m}, 5 \mathrm{H}-\mathrm{Py}), 7.60(1 \mathrm{H}, \mathrm{d}, J=8.5 \mathrm{~Hz}, 5-\mathrm{H}), 7.62(1 \mathrm{H}, \mathrm{m}, 4 \mathrm{H}-\mathrm{Py}), 8.65(1 \mathrm{H}, \mathrm{d}, J=4.4 \mathrm{~Hz}$, $6 \mathrm{H}-\mathrm{Py}) .{ }^{13} \mathrm{C}$ NMR $\left(50 \mathrm{MHz}, \mathrm{CDCl}_{3}\right): \delta 27.7\left(2 \mathrm{xCH}_{3}\right), 55.5\left(\mathrm{OCH}_{3}\right), 76.5(\mathrm{C}-2), 102.9(\mathrm{C}-8)$, 107.0 (C-6), 114.8 (C-3), 123.1 (C-5), 123.6 (C-5 Py), 126.6 (C-3 Py), 129.0 (C-4 Py), 134.1 (C9), 136.7 (C-2 Py), 146.9 (C-10), 155 (C-7), 161.1 (C-4), 165.7 (C-6 Py). MS m/z (\%) 268 (M+): 268 (46), 254 (100), 239 (11), 225 (11), 213 (20), 199 (19), 186 (9), 163 (16), 89 (18).CDCl

\section{2-Ethyl-2-methyl-7-methoxy-3,4-dihydro-4-hydroxy-4-(2-pyridyl)-2H-1-benzopyran-4-one}

5b. Analysis for $\mathrm{C}_{18} \mathrm{H}_{21} \mathrm{NO}_{3}$ (found $\mathrm{C} 71.78, \mathrm{H} \mathrm{6.91,} \mathrm{N} \mathrm{4.57 \% ,} \mathrm{requires} \mathrm{C} 72.20, \mathrm{H} \mathrm{7.07,} \mathrm{N}$ 4.68\%). IR: 3300, 2900, 2360, 1590, 1500, 1440, 1380, 1340, 1260, 1200, 1160, 1140, 1110, 1080, 1030, 980, 935, 880, 840, 795, $745 \mathrm{~cm}^{-1}{ }^{1} \mathrm{H}$ NMR $\left(200 \mathrm{MHz}, \mathrm{CDCl}_{3}\right): \delta 0.93$ (3H, t, $J=$ 6.5Hz, $\left.\mathrm{CH}_{2} \mathrm{CH}_{3}\right), 1.30\left(3 \mathrm{H}, \mathrm{s}, \mathrm{CH}_{3}\right), 1.90\left(2 \mathrm{H}, \mathrm{q}, J=6.5 \mathrm{~Hz}, \mathrm{CH}_{3} \mathrm{CH}_{2}\right), 2.23\left(2 \mathrm{H}, \mathrm{s}, \mathrm{C}_{-} \mathrm{CH}_{2}-\mathrm{C}\right)$, $3.76\left(3 \mathrm{H}, \mathrm{s}, \mathrm{OCH}_{3}\right), 6.33(1 \mathrm{H}, \mathrm{d}, J=2.5 \mathrm{~Hz}, 8-\mathrm{H}), 6.41(1 \mathrm{H}, \mathrm{dd}, J=8.5 \mathrm{~Hz}, 2.5 \mathrm{~Hz}, 6-\mathrm{H}), 6.67(1 \mathrm{H}$, d, $J=8.5 \mathrm{~Hz}, 5-\mathrm{H}), 6.98-7.4$ (2H, m, 3,5H-Py), 7.48-6.93 (1H , m,4H-Py) $8.58(1 \mathrm{H}, \mathrm{d}, J=4.5 \mathrm{~Hz}$, 6H-Py). ${ }^{13} \mathrm{C}$ NMR (50 MHz, $\left.\mathrm{CDCl}_{3}\right): \delta 8.3\left(-\mathrm{CH}_{3}\right), 22.5\left(-\mathrm{CH}_{3}\right), 29.4\left(-\mathrm{CH}_{2}\right), 48.8(\mathrm{C}-3), 55.5(-$ $\mathrm{OCH}_{3}$ ), 70.89 (C-2), 78.14 (C-4), 102.0 (C-8), 108.7 (C-6), 119.7 (C-9), 121.7 (C-5), 122.4 (C-5 Py), 130.8 (C-3 Py), 137.4 (C-4 Py), 146.8 (C-2 Py), 155.6 (C-10), 156.7 (C-4), 166.0 (C-7), 
165.0 (C-6 Py). MS m/z (\%) 299 (M+): 299 (5), 281 (13), 253 (19), 252 (49), 229 (56), 221 (97), 214 (26), 214 (8), 191 (18), 175 (11), 151 (100), 124 (6), 122 (11), 106 (43), 95 (16),79 (45), 63 (7).

2-Ethyl-2-methyl-7-methoxy-4-(2-pyridyl)-2H-1-benzopyran 6b. Analysis for $\mathrm{C}_{18} \mathrm{H}_{19} \mathrm{O}_{2} \mathrm{~N}$ as (found C 76.33, H 6.61, N 4.90\%, requires C 76.83, H 6.81, N 4.98\%). IR(KBr):2900, 1615, 1595, 1575, 1500, 1460, 1440, 1360, 1320, 1280, 1200, 1160, 1135, 1040, 1000, 880, 840, 800, 780, $740 \mathrm{~cm}^{-1},{ }^{1} \mathrm{H}$ NMR (200 MHz, $\left.\mathrm{CDCl}_{3}\right): \delta 1.03\left(3 \mathrm{H}, \mathrm{t}, J=6 \mathrm{~Hz},-\mathrm{CH}_{2} \mathrm{CH}_{3}\right.$ ), $1.46\left(3 \mathrm{H}, \mathrm{s}, \mathrm{CH}_{3}\right), 1.83$ $\left(2 \mathrm{H}, \mathrm{q}, J=6 \mathrm{~Hz}, \mathrm{CH}_{2} \mathrm{CH}_{3}\right), 3.76\left(3 \mathrm{H}, \mathrm{s},-\mathrm{OCH}_{3}\right), 5.80(1 \mathrm{H}, \mathrm{s}, 3-\mathrm{H}), 6.38(1 \mathrm{H}, \mathrm{d}, J=2.0 \mathrm{~Hz}, 6-\mathrm{H})$, $6.56(1 \mathrm{H}, \mathrm{dd}, \mathrm{J}=8.5 \mathrm{~Hz} \& 2.5 \mathrm{~Hz}, 8-\mathrm{H}), 7.23(1 \mathrm{H}, \mathrm{d}, J=8 \mathrm{~Hz}, 5-\mathrm{H}), 7.24(1 \mathrm{H}, \mathrm{m}, 5 \mathrm{H}-\mathrm{Py}), 7.38$ (1H, m, 3H-Py), 7.75 (1H, m, 4H-Py), $8.70(1 \mathrm{H}, \mathrm{d}, J=4.5 \mathrm{~Hz}, 6 \mathrm{H}-\mathrm{Py}) .{ }^{13} \mathrm{C} \mathrm{NMR}(50 \mathrm{MHz}$, $\left.\mathrm{CDCl}_{3}\right): \delta 7.96\left(\mathrm{CH}_{3}\right), 24.80\left(\mathrm{CH}_{3}\right), 33.08\left(\mathrm{CH}_{2}\right), 54.84\left(\mathrm{OCH}_{3}\right), 78.4(\mathrm{C}-2), 102.1(\mathrm{C}-8), 106.0$ (C-6), 114.1 (C-3), 122.1 (C-5), 122.9 (C-5 Py), 125.9 (C-3 Py), 127.4 (C-4 Py), 134.0 (C-9), 136.1 (C-2 Py), 149.0 (C-10), 154.7 (C-7), 156.7 (C-4), 160.5 (C-6 Py). MS m/z (\%) 281 (M ): 281 (17), 266 (25), 252 (100), 237 (8), 222 (13), 203 (9), 194 (14), 180 (16), 166 (8), 155 (9), 126 (8), 89 (6), 78 (12).

2,2-Spirocyclopentyl-7-methoxy-4-hydroxy-4-(2-pyridyl)-3,4-dihydro-2H-1-benzopyran 5c. Analysis for $\mathrm{C}_{19} \mathrm{H}_{21} \mathrm{NO}_{3}$ found $\mathrm{C}$ 73.42, $\mathrm{H}$ 6.67, $\mathrm{N}$ 4.77\%, requires C 73.29, H 6.80, N 4.5\%. IR: 2900, 2360, 1615, 1500, 1430, 1375, 1340, 1280, 1260, 1200, 1160, 1140, 1035, 980, 860, 840, $800 \mathrm{~cm}^{-1},{ }^{1} \mathrm{H}$ NMR (200 MHz, $\left.\mathrm{CDCl}_{3}\right): \delta 1.40-2.16\left(8 \mathrm{H}, \mathrm{m}, 4 \mathrm{xCH}_{2}\right), 2.75\left(2 \mathrm{H}, \mathrm{s}, \mathrm{CH}_{2}\right), 3.80$ $\left(3 \mathrm{H}, \mathrm{s}, \mathrm{OCH}_{3}\right), 6.40-6.6(2 \mathrm{H}, \mathrm{m}, 6$ \&8-H), $6.82(1 \mathrm{H}, \mathrm{d}, J=8.5 \mathrm{~Hz}, 5-\mathrm{H}), 7.13-7.47$ (2H, m, 3 \& 5H-Py), 7.53-7.9 (1H, m, 4H-Py),8.70 (1H, d, J= $4.5 \mathrm{~Hz}, 6 \mathrm{H}-\mathrm{Py}) . \quad \delta$ 1.24-1.96 (8H, m, $\left.\mathrm{CH}_{2}\right)$, $2.26\left(2 \mathrm{H}, \mathrm{s}, \mathrm{CH}_{2}\right), 3.83\left(3 \mathrm{H}, \mathrm{s}, \mathrm{OCH}_{3}\right), 6.35(1 \mathrm{H}, \mathrm{d}, J=2.5 \mathrm{~Hz}, 8-\mathrm{H}), 6.56(1 \mathrm{H}, \mathrm{dd}, J=9 \mathrm{~Hz}$ \& $J=2$ Hz, 6-H), 6.81 (1H, d, J=8.5Hz, 5-H), 7.20 (1H,m 5H-Py), 7.40 (1H, m, 3H-Py), 7.73 (1H, m, 4H-Py), 8.70 (1H, d, J=4 Hz, 6H-Py); ${ }^{13} \mathrm{C}$ NMR (50 MHz, $\left.\mathrm{CDCl}_{3}\right): \delta 23.5\left(-\mathrm{CH}_{3}\right), 24.5(-$ $\mathrm{CH}_{2}$ ), 40.0 (- $\left.\mathrm{CH}_{2}\right), 48.6$ (C-3), 55.5 (- $\left.\mathrm{OCH}_{3}\right), 70.89$ (C-2), 78.14 (C-4), 102.0 (C-8), 108.7 (C-6), 119.7 (C-9), 121.7 (C-5), 122.4 (C-5 Py), 130.8 (C-3 Py), 137.4 (C-4 Py), 146.8 (C-2 Py), 155.6 (C-10), 156.7(C-4), 166.0 (C-7), 165.0 (C-6 Py). MS m/z (\%) 311 (M+); 311 (4), 293 (29), 264 (40), 233 (56), 215 (31), 203 (25), 151 (100), 132 (12), 106 (39), 78 (84), 69 (14), 63 (14).

2,2-Spirocyclopentyl-7-methoxy-4-(2-pyridyl)-2H-1-benzopyran 6c. A solution of 5c (3.1 g, $0.01 \mathrm{~mol})$ and $p$-toluenesulphonic acid $(0.1 \mathrm{~g})$ in benzene $(50 \mathrm{ml})$ was refluxed for $3 \mathrm{~h}$ on a water bath. After cooling to room temperature, the reaction mixture was basified with 5\% sodium carbonate solution, washed with water, dried over anhydrous sodium sulphate and finally concentrated under reduced pressure. The residue obtained was purified by silica gel column chromatography using petroleum ether:ethyl acetate (80:20) as eluent to give 6c a gummy mass (2.54 g, yield 87\%). Analysis: found C 76.99, $\mathrm{H} \mathrm{6.50,} \mathrm{N} \mathrm{4.93 \% ;} \mathrm{C}_{19} \mathrm{H}_{19} \mathrm{NO}_{2}$ requires C 77.79, $\mathrm{H}$ 6.53, N 4.77\%. IR: 2900, 2360, 2340, 1605, 1500, 1440, 1370, 1360, 1300, 1260, 120, 1200, 1160, 1145, 1020, 985, 860, 840, $820 \mathrm{~cm}^{-1},{ }^{1} \mathrm{H}$ NMR (200 MHz, $\left.\mathrm{CDCl}_{3}\right): \delta 1.30-2.02$ (8H, bm, $\left.\mathrm{CH}_{2}\right), 3.89$ (3H, s, $\left.\mathrm{OCH}_{3}\right), 5.90(1 \mathrm{H}, \mathrm{s}, 3-\mathrm{H}), 6.43(1 \mathrm{H}, \mathrm{d}, J=2 \mathrm{~Hz}, 8-\mathrm{H}), 6.60(1 \mathrm{H}, \mathrm{dd}, J=8.5$ \& $2.5 \mathrm{~Hz}, 6-\mathrm{H}), 6.90$ (1H, d, J=8.5 Hz, 5-H), 7.25 (1H, m, 5H-Py), 7.43 (1H, m, 3Н-Py), 7.78 $(1 \mathrm{H}, \mathrm{m}, 4 \mathrm{H}-\mathrm{Py}), 8.78(1 \mathrm{H}, \mathrm{d}, J=4.39 \mathrm{~Hz}, 6 \mathrm{H}-\mathrm{Py}) ;{ }^{13} \mathrm{C} \mathrm{NMR}\left(50 \mathrm{MHz}, \mathrm{CDCl}_{3}\right): \delta 20.1\left(-2 \mathrm{XCH}_{2}\right)$, 
$28.3\left(-\mathrm{CH}_{2}\right), 39.3\left(-2 \mathrm{XCH}_{2}\right), 48.6$ (C-3), $55.5\left(-\mathrm{OCH}_{3}\right), 70.89$ (C-2), 102.0 (C-8), 108.7 (C-6), 114.7 (C-3), 119.7 (C-9), 121.7 (C-5), 122.4 (C-5 Py), 130.8 (C-3 Py), 137.4 (C-4 Py), 146.8 (C2 Py), 155.6 (C-10), 156.7(C-4), 166.0 (C-7), 165.0 (C-6 Py); MS m/z (\%) 293 (M+); 293 (22), 258 (76), 225 (52), 195 (39), 143 (63), 124 (48), 106 (22), 71 (18).

2,2-Spirocyclohexyl-7-methoxy-4-hydroxy-4-(2-pyridyl)-3,4-dihydro-2H-1-benzopyran $5 \mathrm{~d}$. Analysis for $\mathrm{C}_{20} \mathrm{H}_{23} \mathrm{O}_{3}$ (found C 74.33, $\mathrm{H}$ 6.87, $\mathrm{N} 4.18 \%$, requires C 73.82, H 7.12, N 4.30\%). IR: 3365, 2850, 1620, 1600, 1500, 1440, 1340, 1280, 1260, 1200, 1160, 1140, 1085, 1040, 985, 840, 785, $745 \mathrm{~cm}^{-1},{ }^{1} \mathrm{H}$ NMR (200 MHz, $\left.\mathrm{CDCl}_{3}\right): \delta 1.45-1.96\left(10 \mathrm{H}, \mathrm{m}, 5 \mathrm{xCH}_{2}\right), 2.26\left(2 \mathrm{H}, \mathrm{s}, \mathrm{C}^{-\mathrm{CH}_{2}}\right)$, $3.83\left(3 \mathrm{H}, \mathrm{s}, \mathrm{OCH}_{3}\right), 6.53-6.66(2 \mathrm{H}, \mathrm{m}, 6$ \&8-H), $6.83(1 \mathrm{H}, \mathrm{d}, J=8.5 \mathrm{~Hz}, 5-\mathrm{H}), 7.0-7.43(2 \mathrm{H}, \mathrm{m}$, 3, 5H-Py), 7.73 (1H, m, 4H-Py), $8.63(1 \mathrm{H}, \mathrm{d}, J=4.5 \mathrm{~Hz}, 6 \mathrm{H}-\mathrm{Py}) .{ }^{13} \mathrm{C}$ NMR $\left(50 \mathrm{MHz}, \mathrm{CDCl}_{3}\right): \delta$ $22.4\left(\mathrm{CH}_{2}\right), 22.5\left(\mathrm{CH}_{2}\right), 26.0\left(\mathrm{CH}_{2}\right), 32.9\left(\mathrm{CH}_{2}\right), 38.9\left(\mathrm{CH}_{2}\right), 50.2\left(-\mathrm{CH}_{2}\right), 55.6\left(-\mathrm{OCH}_{3}\right), 70.9(\mathrm{C}-$ 3), 76.6 (C-4), 102.1 (C-8), 108.8 (C-6), 120.2 (C-9), 122.2 (C-5), 126.5 (C-5 Py), 132.5 (C-3 Py), 138.7 (C-4 Py), 147.8 (C-3), 155.2 (C-10), 160.8 (C-7), 165.9 (C-6 ), MS m/z (\%) 325 (M+); 325 (18), 307 (12), 264 (23), 247 (40), 230 (52), 200 (13), 166 (8), 151 (100), 149 (21), 134 (36), 106 (54), 78(36), 69 (8), 63 (6).

2,2-Spirocyclohexyl-7-methoxy-4-(2-pyridyl)-2H-1-benzopyran 6d. Analysis for $\mathrm{C}_{20} \mathrm{H}_{21} \mathrm{O}_{2} \mathrm{~N}$ (found C 77.93, H 6.76, N 4.49\%, requires C 78.15, H 6.89, N 4.56\%). IR: 2850, 1620, 1600, 1500, 1460, 1440, 1360, 1320, 1280, 1260, 1200, 1160, 1120, 1040, 1000, 985, 920, 840, 810, $795 \mathrm{~cm}^{-1},{ }^{1} \mathrm{H}$ NMR $\left(200 \mathrm{MHz}, \mathrm{CDCl}_{3}\right): \delta 1.33-2.00\left(10 \mathrm{H}, \mathrm{m}, 5 \mathrm{xCH}_{2}\right), 3.87\left(3 \mathrm{H}, \mathrm{s}, \mathrm{OCH}_{3}\right) 5.87$ $(1 \mathrm{H}, \mathrm{s}, 3-\mathrm{H}), 6.40(1 \mathrm{H}, \mathrm{d}, J=2.5 \mathrm{~Hz}, 8-\mathrm{H}), 6.63(1 \mathrm{H}, \mathrm{dd}, J=8.5 \& 2.5 \mathrm{~Hz}, 6-\mathrm{H}), 7.20(1 \mathrm{H}, \mathrm{d}, J=$ 8.5Hz, 5-H), $7.32(1 \mathrm{H}, \mathrm{m}, 5 \mathrm{H}-\mathrm{Py}), 7.45(1 \mathrm{H}, \mathrm{m}, 3 \mathrm{H}-\mathrm{Py}), 7.68(1 \mathrm{H}, \mathrm{m}, 4 \mathrm{H}-\mathrm{Py}), 8.57(1 \mathrm{H}, \mathrm{d}$, $\mathrm{J}=4.4 \mathrm{~Hz}, 6 \mathrm{H}-\mathrm{Py}),{ }^{13} \mathrm{C}$ NMR(50 MHz, $\left.\mathrm{CDCl}_{3}\right): \delta 22.4\left(\mathrm{CH}_{2}\right), 22.5\left(\mathrm{CH}_{2}\right), 26.0\left(\mathrm{CH}_{2}\right), 32.9$ $\left(\mathrm{CH}_{2}\right), 38.9\left(\mathrm{CH}_{2}\right), 50.2\left(-\mathrm{CH}_{2}\right), 55.6\left(-\mathrm{OCH}_{3}\right), 102.1(\mathrm{C}-8), 108.8$ (C-6), 114.6 (C-3), $120.2(\mathrm{C}-$ 9), 122.2 (C-5), 126.5 (C-5 Py), 132.5 (C-3 Py), 138.7 (C-4 Py), 147.8 (C-3), 155.2 (C-10), 157.2 (C-4), 160.8 (C-7), 165.9 (C-6 ), MS m/z (\%) $306\left(\mathrm{M}^{+}\right)$; 306 (46), 278 (18), 263 (100), 250 (35), 228 (10), 214 (19), 192 (15), 180 (16), 166 (15), 153 (13), 79 (14), 77 (18), 52 (19).

\section{2,2-Dimethyl-4-hydroxy-6-nitro-7-methoxy-3,4-dihydro-4-(2-pyridyl)-2H-1-benzopyran}

(5e). Analysis for $\mathrm{C}_{17} \mathrm{H}_{18} \mathrm{~N}_{2} \mathrm{O}_{5}$ (found $\mathrm{C} 60.27, \mathrm{H}$ 5.51, N 8.33\%, requires C 61.81, H 5.49, N 8.48\%). IR(KBr): 3275, 2940, 1590, 1515, 1440, 1390, 1370, 1330, 1250, 1240, 1210, 1150, 1120, 1060, 1030, 980, 940, 840, 780, 750, $730 \mathrm{~cm}^{-1}{ }^{1} \mathrm{H} \mathrm{NMR}\left(\mathrm{CDCl}_{3}\right): 1.48$ (3H, s,2xCH$), 2.66$ $\left(2 \mathrm{H}, \mathrm{s}, \mathrm{CH}_{2}\right), 3.98\left(3 \mathrm{H}, \mathrm{s}, \mathrm{OC} \mathrm{H}_{3}\right), 6.26(1 \mathrm{H}, \mathrm{s}, 8-\mathrm{H}), 7.23(1 \mathrm{H}, \mathrm{s}, 5-\mathrm{H}), 7.30(1 \mathrm{H}, \mathrm{dd}, \mathrm{J}=2.2$ \& $\left.8.2 \mathrm{~Hz}, 3^{`}-\mathrm{H}\right), 7.41\left(1 \mathrm{H}, \mathrm{m}, 5^{`}-\mathrm{H}\right), 7.93\left(1 \mathrm{H}, \mathrm{m}, 4^{`}-\mathrm{H}\right), 8.65\left(1 \mathrm{H}, \mathrm{dd}, \mathrm{J}=2.2 \& 8.2 \mathrm{~Hz}, 6^{`}-\mathrm{H}\right) .{ }^{13} \mathrm{C}$ NMR (50 MHz, CDCl $): \delta 25.8\left(\mathrm{CH}_{3}\right), 25.9\left(\mathrm{CH}_{3}\right), 51.6(\mathrm{C}-3), 55.9\left(\mathrm{OCH}_{3}\right), 72.1(\mathrm{C}-2), 75.8$ (C-4), 103.0 (C-8), 116.9 (C-6), 121.9 (C-9), 121.2 (C-5), 122.6 (C-5 Py), 131.7 (C-3 Py), 138.1 (C-4 Py), 147.0 (C-2 Py), 156.4 (C-10), 159.2 (C-7), 166.2 (C-6 Py). MS: m/z 330(18), 328(55), 312(100), 267(22), 276(17), 255(10), 240(5), 234(14), 204(11), 188(9), 160(7), 133(13), 107(4). 2,2-Dimethyl-6-nitro-7-methoxy-4-(2-pyridyl)-2H-1-benzopyran 6e. Analysis for $\mathrm{C}_{17} \mathrm{H}_{16} \mathrm{~N}_{2} \mathrm{O}_{4}$ (found C 65.17, H 5.20, N 8.83\%, requires C 65.38, H 5.16, N 8.99\%). IR: 3270, 2940, 1580, 1520, 1440, 1390, 1360, 1330, 1250, 1230, 1210, 1140, 1120,1060, 1020, 980, 930, 840, 770, 750, 735 $\mathrm{cm}^{-1}{ }^{1} \mathrm{H} \mathrm{NMR}\left(\mathrm{CDCl}_{3}\right): 1.48\left(6 \mathrm{H}, \mathrm{s}, 2 \mathrm{xCH}_{3}\right), 3.92\left(3 \mathrm{H}, \mathrm{s}, \mathrm{OCH}_{3}\right), 6.30(1 \mathrm{H}, \mathrm{s}, 3-\mathrm{H}) 6.92$ 
(1H, s, 8-H), 7.72 (1H, s, 5-H), 7.25 (1H, dd, J=2.2 \& 8.2Hz, 3`-H) , 7.35 (1H, m, 5`-H), 7.95 $\left(1 \mathrm{H}, \mathrm{m}, 4{ }^{`}-\mathrm{H}\right), 8.65\left(1 \mathrm{H}, \mathrm{dd}, \mathrm{J}=2.2 \& 8.2 \mathrm{~Hz}, 6{ }^{`}-\mathrm{H}\right) .{ }^{13} \mathrm{C}$ NMR $\left(50 \mathrm{MHz}, \mathrm{CDCl}_{3}\right): \delta 28.1$ (2xCH 3$), 55.8\left(\mathrm{OCH}_{3}\right), 77.3$ (C-2), 103.8 (C-8), 112.9 (C-6), 115.3 (C-3), 120.8 (C-5), 124.5 (C5 Py), 127.2 (C-3 Py), 129.4 (C-4 Py), 136.2 (C-9), 138.0 (C-2 Py), 147.0 (C-10), 156.3 (C-7), 161.9 (C-4), 166.6 (C-6 Py). MS: $\mathrm{M}^{+}$at $\mathrm{m} / \mathrm{z}$ 312(48), 310(32), 294(22), 248(100), 267(12), 238(20), 222(7), 194(15), 170(17), 142(1), 115(18), 89(7).

\section{Acknowledgements}

Authors are thankful to CSIR, Delhi for the project grant.

\section{References}

1. Gopalakrishnan, M.; Jainis, R. A.; Triggle, D. J. Drug Dev. Res. 1993, $28,95$.

2. Li H-Y, Hu. C. Chin. Pharm. J. Beijing 2005, 40, 240.

3. Ellis, G. P.; Lochhart, I. M., Eds, Chemistry of Heterocyclic Compounds; Chromans and Tocopherols, Wiley: New York, N. Y., 1981; Vol. 36, p 469.

4. Vicario, J. L.; Badia, D.; Carrillo, L. Tetrahedron: Asymmetry 2003, 14, 489.

5. Lloyd, J.; Atwal, K. S.; Finlay. H. J.; Nyman. M.; Huynh, T.; Rao, B.; Kover, A.; Schmidt, J.; Vaccaro, W.; Conder, M. L.; West, T. J.; Levesque, P. Bioorg. Med. Chem. Lett. 2007, 17, 3271.

6. Francisco, P.; Barros, A. I. R. N. A.; Artur Silva, M. S. J. Biochem. Mol. Toxicology 2002, 16, 220.

7. Edwards, G.; Weston, A. H. Trends Pharm. Sci. 1990, 11, 417.

8. Joshi, S. S.; Singh, H. J. Am. Chem. Soc. 1954, 76, 4993.

9. Kabbe, H. J. Chem. Soc. Rev. 1997, 26, 233.

10. Ashwood, A. V.; Buckingham, R. E.; Cassidy, F.; Evans, J. M.; Faruk, E. A.; Hamilton, T. C.; Nash, D. J.; Willcocks, G.; Stemp, K. J. Med. Chem. 1986, 29, 2194.

11. Emmert, B. Chem. Ber. 1939, 72b, 1188.

12. Monks, A.; Scudiero, D.; Skehan, P.; Shoemaker, R.; Paull, K.; Vistica, D.; Hose, C.; Langley, I.; Cronise, P.; Vaigro-Wolff, A.; Gray-Goodrich, M.; Campbell, H.; Mayo, J.; Boyd, M. J. Natl. Cancer Inst. 1991, 83, 757.

13. Debatin, K. M. Toxicol. Lett. 2000, 112/113, 41.

14. Yoon, Y.; Kim, Y. O.; Lim, N. Y.; Leon, W. Y.; Sung, H. J. Planta Medica 1999, 65, 532.

15. Kerr, J. F. R.; Wyllie, H. A.; Currie, A. R. Br. J. Cancer 1972, 26, 239.

16. Dini, L. Tissue Cell. 2005, 37, 379.

17. Rello, S.; Stockert, J. C.; Moreno, V.; Gamez, A.; Pacheco, M.; Juarranz, A.; Canete, M.; Villanueva, A. Apoptosis 2005, 10, 201. 
18. Ellis, G. P.; Chemistry of Heterocyclic Compounds; Chromenes, chromanones and chromones, Eds ,Wiley: New York, N. Y. 1977; Vol. 31, p 1196.

19. Cassidy, F.; Evans, J. M.; Smith, D. M.; Stemp, G.; Edge, C.; Williams, D. J. Chem. Soc., Chem. Commun. 1989, 377.

20. Bergmann, R.; Gericke. R. J. Med. Chem. 1990, 33, 492.

21. Sassen, L. M. A.; DunKer, D. J. G. M.; Gho, B. C. G. Br. J. Pharmacol. 1990, 101, 605.

22. Quagliato, D. A.; Humber, L. G. EPA 0314446, 1989; Chem. Abstr. 1990, 112, $48798 \mathrm{z}$.

23. Garcia, G.; Di Malta. A.; Soubrie, P. EPA 0370901, 1990; Chem. Abstr. 1990, 113, 191161n.

24. (a) Attwood, M. R.; Churcher, I.; Dunsdon, R. M.; Hurst, D. N.; Jones, P. S. Tetrahedron Lett. 1991, 32, 811. (b) Attwood, M. R.; Jones, P. S.; Kay, P. B.; Paciorek, P. M.; Redshaw, S. Life Sci. 1991, 48, 803.

25. Ritter, K. Synthesis 1993, 735.

26. Kalinin, V. N. Synthesis 1992, 413.

27. Tripathi, A. K.; Khan, K. R.; Taneja, S. C. Synth. Commun. 2003, 33, 579.

28. Tripathi, A. K.; Koul, S.; Taneja, S. C. Ind. J. Chem. 2009, 48B, 301.

29. Emmert B.; Asendorf, E. Chem. Ber. 1939, 72b, 1188. 